\title{
Targeted next-generation sequencing to assess tumor mutation burden: ready for prime-time in non-small cell lung cancer?
}

\author{
Pascale Tomasini ${ }^{1,2}$, Laurent Greillier ${ }^{1,2}$ \\ ${ }^{1}$ Department of Multidisciplinary Oncology and Therapeutic Innovations, Assistance Publique Hopitaux de Marseille, Aix-Marseille University, \\ Marseille, France; ${ }^{2}$ Centre de Recherche en Cancérologie de Marseille (CRCM), Marseille, France \\ Correspondence to: Prof. Laurent Greillier, MD, PhD. Multidisciplinary Oncology \& Therapeutic Innovations Department. Hôpital Nord, Chemin \\ des Bourrely, 13015 Marseille, France. Email: laurent.greillier@ap-hm.fr. \\ Provenance: This is an invited article commissioned by the Section Editor Hengrui Liang (Department of Thoracic Surgery, Guangzhou Medical \\ University, Guangzhou, China). \\ Comment on: Rizvi H, Sanchez-Vega F, La K, et al. Molecular Determinants of Response to Anti-Programmed Cell Death (PD)-1 and Anti- \\ Programmed Death-Ligand 1 (PD-L1) Blockade in Patients With Non-Small-Cell Lung Cancer Profiled With Targeted Next-Generation \\ Sequencing. J Clin Oncol 2018;36:633-41.
}

Submitted Jun 30, 2019. Accepted for publication Sep 01, 2019.

doi: $10.21037 /$ tlcr.2019.09.01

View this article at: http://dx.doi.org/10.21037/tlcr.2019.09.01

Non-small cell lung cancer (NSCLC) treatment strategy has recently evolved with the development of immune checkpoint inhibitors (ICIs). Several ICIs have indeed been approved for the treatment of locally advanced and metastatic NSCLC on the basis of phase III trials. Two monoclonal antibodies directed to programmed death-1 (PD-1), namely nivolumab $(1,2)$ and pembrolizumab (3), and one antibody directed to programmed death-ligand 1 (PD-L1), namely atezolizumab (4), have shown longer overall survival (OS) than standard chemotherapy in previously treated patients with advanced NSCLC. Pembrolizumab also showed longer OS than platinumbased chemotherapy for the first-line treatment of advanced NSCLC alone (5) or in combination with platinumbased chemotherapy $(6,7)$. Moreover, the PD-L1 inhibitor durvalumab was approved after chemoradiotherapy in patients with unresectable locally advanced NSCLC $(8,9)$. Nevertheless, despite OS benefit, the response rate with ICIs in unselected population is low (approximately 20\%), suggesting ICIs are not effective for every NSCLC patient. There is thus a need for the identification of predictive biomarkers to select patients with the highest probability to derive benefit from ICIs.

PD-L1 expression was the first assessed biomarker for prediction of ICIs' efficacy and pembrolizumab single agent is only approved for the second-line treatment of
PD-L1 positive NSCLC (3) or for the first-line treatment of NSCLC expressing $\geq 50 \%$ PD-L1 (5). However, $\mathrm{PD}-\mathrm{L} 1$ expression is a continuous variable and PD-L1 immunohistochemistry assessment by pathologists is difficult, with a poor inter-observer reproducibility (10). In addition, there is a spatial and temporal heterogeneity and PD-L1 expression results can vary depending on the area and the time where the biopsy is performed (10). Moreover, multiple assays, platforms and cut-offs where developed to identify companion diagnostic test in the princeps phase III studies of ICIs. Despite the efforts made towards standardization and harmonization of assays, especially with the Blue print 1 and 2 working groups $(11,12)$, PD$\mathrm{L} 1$ is not a perfect biomarker and additional biomarkers are eagerly awaited to better predict ICIs' efficacy.

The most studied biomarker after PD-L1 expression is tumor mutation burden (TMB). TMB is defined as the number of mutations per DNA megabases. TMB was measured at particularly high level in solid tumors known to be sensitive to ICIs such as NSCLC, melanoma or bladder cancer (13). TMB association with ICIs efficacy in advanced NSCLC patients was studied in several clinical trials. In the CheckMate 227 trial, progression-free survival (PFS) was longer with a combination of nivolumab and ipilimumab compared with first-line chemotherapy in tumors with TMB $\geq 10$ mut/Mb (14). In the OAK trial, the PFS difference 
between atezolizumab and docetaxel was larger in patients with blood TMB $\geq 16$ mut/Mb (15). In the MYSTIC trial, there was a non-statistically significant advantage in terms of OS with durvalumab +/- tremelimumab in patients with tissue TMB $\geq 10$ mut/Mb (16). A blood TMB $\geq 20 \mathrm{mut} / \mathrm{Mb}$ was associated with statistically improved survival with durvalumab +/- tremelimumab in comparison with chemotherapy. In these trials, PD-L1 expression and TMB were independent biomarkers.

TMB is ideally evaluated using whole genome sequencing (WGS) or at least whole exome sequencing (WES). However, WGS and WES are not ready for daily practice use because they are long, expensive and need a high quantity of tumor DNA. TMB can also be assessed with targeted NGS (17). However, there is a lack of data regarding the ideal panel and cut-off to use for TMB assessment.

In 2018, Rizvi and colleagues published in the Fournal of Clinical Oncology a study primarily aimed to determine the potential of TMB assessed with targeted NGS to predict ICIs' efficacy in NSCLC patients (18). Secondary objectives were to examine the correlation of TMB derived with WES and targeted NGS in a subset of tumors, to determine the potential of copy number alterations (CNA) and specific genes mutations to predict ICIs efficacy and to assess the relationship between TMB and PD-L1 expression.

Clinical, biological, treatment and outcome data were retrieved from medical records of patients with advanced NSCLC treated with ICIs between April 2011 and January 2017 who had a tumor molecular profile performed by MSK-IMPACT (18). The authors identified also an independent cohort of advanced NSCLC patients, who were not treated with ICI, but with MSK-IMPACT molecular profiling performed. All patients $(\mathrm{N}=240)$ underwent MSKIMPACT targeted NGS with a panel of 341 to 468 genes (covering 0.98 to $1.22 \mathrm{Mb}$ ), depending on the version used. The samples were collected before immunotherapy for $85 \%$ of patients. WES was also performed in a subgroup of patients $(\mathrm{N}=49)$. The same tumor sample was used for NGS and WES for 40 patients. PD-L1 expression was assessed with several antibodies (E1L3N, Cell signaling; 28-8, DAKO; 22C3, DAKO) in 84 tumors.

There was a good correlation between TMB assessed by targeted NGS and TMB assessed by WES (Spearman $\mathrm{r}=0.86 ; \mathrm{P}=0.001)(18)$. TMB was associated with ICIs efficacy. Patients with TMB above the $50^{\text {th }}$ percentile had better durable clinical benefit (DCB rate, $38.6 \%$ vs. $25.1 \%$; $\mathrm{P}=0.009$ ) and longer PFS (PFS HR, 1.38; $\mathrm{P}=0.024)$ than patients with TMB below the $50^{\text {th }}$ percentile. The fraction of CNA was lowest in patients with DCB and significantly higher in patients with no durable benefit than patients who did not receive ICIs (0.16 vs. $0.11 ; \mathrm{P}=0.007)$. EGFR and STK11 mutations were associated with no durable benefit $(\mathrm{P}=0.013$ and $\mathrm{P}=0.007$ respectively). Finally, whereas PD-L1 expression was associated with longer PFS (HR, $0.526 ; \mathrm{P}=0.011)$, there was no correlation between $\mathrm{PD}$ L1 expression and TMB (Spearman $r=0.1915 ; \mathrm{P}=0.08$ ) and PD-L1 expression and the fraction of CNA (Spearman $\mathrm{r}=-0.1273 ; \mathrm{P}=0.25)$.

TMB seems to be useful to select NSCLC patients for treatment with ICIs. However, implementing the TMB assessment in daily clinical practice is a real challenge for several reasons $(19,20)$. First, the test has to be performed on routinely obtained tumor samples. In patients with advanced NSCLC, biopsy specimens are usually small, with consequently a small quantity of DNA. Moreover, the results must be provided within a limited time frame and must facilitate treatment decisions. Finally, the cost of the test must be reasonable.

For these reasons, while WES is generally considered as the gold standard for TMB measurement, its use is not compatible with routine clinical practice in oncology. Therefore, TMB measurement using targeted NGS panels has been examined (20). With quicker turnaround time, lesser DNA input requirements and lower sequencing costs, targeted NGS may fit the clinical practice requirements. Results from Rizvi and colleagues showed that TMB assessment with their "home-made" targeted NGS is reliable compared with WES (18). Concordant results have been obtained with other platforms, such as the commercially available Foundation One assay. Chalmers and colleagues showed in a cohort of 29 tumors that TMB calculations by either targeted NGS (with the Foundation One assay targeting approximately $1.1 \mathrm{Mb}$ of coding genome and 315 genes) or WES were highly correlated (17). The authors also showed that sequencing genome fractions inferior to $0.5 \mathrm{Mb}$ resulted in unacceptable variation in TMB estimation compared with WES, advising that targeted NGS with narrower sequencing may incorrectly estimate the TMB.

However, the dependability of targeted NGS panels to accurately predict response to ICIs encounters several challenges and numerous questions have to be answered before a definitive transfer into clinical routine practice (21). For example, what is the optimal size of NGS panels? Should we prefer commercially available assays or "homemade" testing? What is optimal cutoff value to define high 
TMB? In addition, a robust analytical validation is needed, while the turnaround time is still relatively long. Finally, tests must be easily accessible for patients and caregivers and the costs have to be reasonable before routine testing could be performed at a large scale. Recently, efforts have been done towards an optimization and harmonization of TMB measurement, including mathematical modeling and bioinformatic pipelines to help TMB quantification (22).

Another point of discussion is related to the predictive or prognostic value of TMB. As Rizvi and colleagues did not observe a positive correlation between TMB and OS in a cohort of patients not treated with ICIs, they concluded that TMB was a predictive biomarker, and not a prognostic one (18). From a methodological point of view, independent data from randomized studies, comparing ICIs to nonICIs treatment, are preferable to demonstrate the purely predictive value of TMB in NSCLC patients. Fortunately, there is growing number of phase III clinical trials incorporating TMB assessment in the study design.

While TMB looks like a promising predictive biomarker for ICIs efficacy, it will certainly not completely replace PDL1 assessment in the real-world setting. Neither TMB nor PD-L1 is fully sensitive or specific of the outcomes. Rizvi and colleagues showed that PD-L1 expression and TMB were independent variables, both associated with ICIs' efficacy (18). TMB looks as good as PD- L1 expression to predict clinical outcomes, but combination of both variables may be even more meaningful to select NSCLC patients that are the most likely to derive a clinical benefit from treatment. The addition of other potential biomarkers, such as CNA, single gene alterations or molecular signatures, to better predict the effectiveness of ICIs in NSCLC patients is another burning question. Furthermore, mathematical modeling, integrating an exponential number of data coming from genomics, transcriptomics, proteomics and immunomics, may be useful in the future of precision oncology.

But will we still use tumor samples in the next years to select treatment in NSCLC patients? With the development of liquid biopsy, another promising approach is the measurement of TMB in cell-free DNA (cfDNA) in peripheral blood. Khagi and colleagues assessed 69 patients with different malignancies who received ICIs and bloodderived circulating tumor DNA (ctDNA) NGS testing (23). They reported a significant association between the number of alterations on liquid biopsy and ICIs outcomes. Koeppel and colleagues measured TMB in cfDNA isolated from blood of 32 patients with several metastatic diseases and compared the results with TMB assessment using tissue
WES (24). They reported a sensitivity of $53 \%$, which is quite low. This might be explained by the fact ctDNA was negative or mildly positive in some patients' blood samples. This observation suggests that the quantity of ctDNA is a critical parameter for TMB evaluation in the blood and could be the Achilles' heel of this promising approach from a pragmatic clinical point of view.

In conclusion, evaluation of the TMB may be useful in the near future to guide patients' selection for ICIs therapy. TMB assessment with targeted NGS appears to be feasible and compatible with the requirements of daily clinical practice in oncology. However, there is a lot of questions to be answered before this test could be implemented in routine practice. Furthermore, TMB measurement with targeted NGS has to be standardized to ensure reliability, reproducibility and clinical usefulness of this biomarker (20).

\section{Acknowledgments}

None.

\section{Footnote}

Conflicts of Interest: The authors have no conflicts of interest to declare.

Ethical Statement: The authors are accountable for all aspects of the work in ensuring that questions related to the accuracy or integrity of any part of the work are appropriately investigated and resolved.

\section{References}

1. Borghaei H, Paz-Ares L, Horn L, et al. Nivolumab versus Docetaxel in Advanced Nonsquamous Non-Small-Cell Lung Cancer. N Engl J Med 2015;373:1627-39.

2. Brahmer J, Reckamp KL, Baas P, et al. Nivolumab versus Docetaxel in Advanced Squamous-Cell Non-Small-Cell Lung Cancer. N Engl J Med 2015;373:123-35.

3. Herbst RS, Baas P, Kim DW, et al. Pembrolizumab versus docetaxel for previously treated, PD-L1-positive, advanced non-small-cell lung cancer (KEYNOTE-010): a randomised controlled trial. Lancet 2016;387:1540-50.

4. Rittmeyer A, Barlesi F, Waterkamp D, et al. Atezolizumab versus docetaxel in patients with previously treated non-small-cell lung cancer (OAK): a phase 3, openlabel, multicentre randomised controlled trial. Lancet 2017;389:255-65. 
5. Reck M, Rodríguez-Abreu D, Robinson AG, et al. Pembrolizumab versus Chemotherapy for PD-L1Positive Non-Small-Cell Lung Cancer. N Engl J Med 2016;375:1823-33.

6. Gandhi L, Rodríguez-Abreu D, Gadgeel S, et al. Pembrolizumab plus Chemotherapy in Metastatic NonSmall-Cell Lung Cancer. N Engl J Med 2018;378:2078-92.

7. Paz-Ares L, Luft A, Vicente D, et al. Pembrolizumab plus Chemotherapy for Squamous Non-Small-Cell Lung Cancer. N Engl J Med 2018;379:2040-51.

8. Antonia SJ, Villegas A, Daniel D, et al. Durvalumab after Chemoradiotherapy in Stage III Non-Small-Cell Lung Cancer. N Engl J Med 2017;377:1919-29.

9. Antonia SJ, Villegas A, Daniel D, et al. Overall Survival with Durvalumab after Chemoradiotherapy in Stage III NSCLC. N Engl J Med 2018;379:2342-50.

10. McLaughlin J, Han G, Schalper KA, et al. Quantitative Assessment of the Heterogeneity of PD-L1 Expression in Non-Small-Cell Lung Cancer. JAMA Oncol 2016;2:46-54.

11. Hirsch FR, McElhinny A, Stanforth D, et al. PDL1 Immunohistochemistry Assays for Lung Cancer: Results from Phase 1 of the Blueprint PD-L1 IHC Assay Comparison Project. J Thorac Oncol 2017;12:208-22.

12. Tsao MS, Kerr KM, Kockx M, et al. PD-L1 Immunohistochemistry Comparability Study in Real-Life Clinical Samples: Results of Blueprint Phase 2 Project. J Thorac Oncol 2018;13:1302-11.

13. Alexandrov LB, Nik-Zainal S, Wedge DC, et al. Signatures of mutational processes in human cancer. Nature 2013;500:415-21.

14. Hellmann MD, Ciuleanu TE, Pluzanski A, et al. Nivolumab plus Ipilimumab in Lung Cancer with a High Tumor Mutational Burden. N Engl J Med 2018;378:2093104.

15. Gandara DR, Kowanetz M, Mok TSK, et al. Blood-based biomarkers for cancer immunotherapy: Tumor mutational burden in blood (bTMB) is associated with improved atezolizumab (atezo) efficacy in 2L+ NSCLC (POPLAR

Cite this article as: Tomasini P, Greillier L. Targeted nextgeneration sequencing to assess tumor mutation burden: ready for prime-time in non-small cell lung cancer? Transl Lung Cancer Res 2019;8(Suppl 4):S323-S326. doi: 10.21037/ tlcr.2019.09.01 and OAK). Ann Oncol 2017;28:v460-96.

16. Peters S, Cho BC, Reinmuth N, et al. Tumor Mutational Burden (TMB) as a Biomarker of Survival in Metastatic NSCLC (mNSCLC): Blood and Tissue TMB Analysis from MYSTIC, a Phase 3 Study of First-line Durvalumab \pm Tremelimumab vs Chemotherapy. Cancer Res 2019. doi: 10.1158/1538-7445.AM2019-CT074

17. Chalmers ZR, Connelly CF, Fabrizio D, et al. Analysis of 100,000 human cancer genomes reveals the landscape of tumor mutational burden. Genome Med 2017;9:34.

18. Rizvi H, Sanchez-Vega F, La K, et al. Molecular Determinants of Response to Anti-Programmed Cell Death (PD)-1 and Anti-Programmed Death-Ligand 1 (PD-L1) Blockade in Patients With Non-Small-Cell Lung Cancer Profiled With Targeted Next-Generation Sequencing. J Clin Oncol 2018;36:633-41.

19. Truesdell J, Miller VA, Fabrizio D. Approach to evaluating tumor mutational burden in routine clinical practice. Transl Lung Cancer Res 2018;7:678-81.

20. Meléndez B, Van Campenhout C, Rorive S, et al. Methods of measurement for tumor mutational burden in tumor tissue. Transl Lung Cancer Res 2018;7:661-7.

21. Ilie M, Hofman P. Spotlight on tumor mutational burden in patients with non-small cell lung carcinoma. Transl Lung Cancer Res 2018;7:614-5.

22. Fancello L, Gandini S, Pelicci PG, et al. Tumor mutational burden quantification from targeted gene panels: major advancements and challenges. J Immunother Cancer 2019;7:183.

23. Khagi Y, Goodman AM, Daniels GA, et al. Hypermutated Circulating Tumor DNA: Correlation with Response to Checkpoint Inhibitor-Based Immunotherapy. Clin Cancer Res 2017;23:5729-36.

24. Koeppel F, Blanchard S, Jovelet C, et al. Whole exome sequencing for determination of tumor mutation load in liquid biopsy from advanced cancer patients. PLoS One 2017;12:e0188174. 